\title{
AMOSTRAGEM DE FORMIGAS-CORTADEIRAS (HYMENOPTERA: FORMICIDAE) EM EUCALIPTAIS PELOS MÉTODOS DE TRANSECTOS EM FAIXA E EM LINHA ${ }^{1}$
}

\author{
Marcelo de Almeida Reis², Ronald Zanetti ${ }^{3}$, José Roberto Soares Scolforo ${ }^{3}$ e Maria Zélia Ferreira ${ }^{4}$
}

RESUMO - Este trabalho foi realizado em eucaliptais localizados em áreas de relevo acidentado da Mata Atlântica, no Município de Belo Oriente, Vale do Rio Doce, Minas Gerais, no período de janeiro de 2002 a fevereiro de 2005, em cinco talhões de Eucalyptus spp., totalizando 160,22 ha. Os objetivos foram desenvolver planos de amostragem para formigas-cortadeiras pelo método de transectos em faixas; determinar o melhor estimador de densidade populacional de formigueiros para os métodos de transectos em faixa e em linha; e validar o melhor plano de amostragem. Em cada entrelinha de plantio de cada talhão, os ninhos de formigas-cortadeiras foram localizados, georreferenciados e medidos. Foram gerados mapas com a localização dos ninhos e das árvores nos talhões, que serviram para a simulação dos planos. Concluiu-se que qualquer uma das linhas testadas para o lançamento de primeiro transecto pode ser utilizada no monitoramento de formigas-cortadeiras na área de estudo. A distância ótima entre os transectos em faixa foi de $96 \mathrm{~m}$. A estimativa da densidade de ninhos de formigas-cortadeiras pelo estimador de área proporcional, usando transectos em faixa, é mais precisa do que pelo estimador Cottam \& Curtis, com a utilização de transectos em linha, pois o primeiro produz resultados iguais aos do censo. O uso operacional dos transectos em faixa, com o estimador de área proporcional, lançados a cada 96 m a partir da terceira linha de plantio é valido para estimar a área total de formigueiros na região.

Palavras-chave: Monitoramento, Formicidae, Eucalyptus spp.

\section{SAMPLING PLANS FOR LEAF-CUTTING ANT NESTS (HYMENOPTERA: FORMICIDAE) BY THE METHODS OF STRIP TRANSECTS AND LINE TRANSECTS ON EUCALYPTUS PLANTATIONS}

\begin{abstract}
The present work was accomplished in eucalyptus plantations, in the municipality of Belo Oriente, Vale do Rio Doce, Minas Gerais, during the period of January of 2002 to February of 2005, in five Eucalyptus spp. stands, totaling 160.22ha. The objectives were to develop sampling plans by the method of strip transects; determine the best estimator of ant nests for the methods of strip and line transects and validate the best sampling plan. In each interrow, the ant nests were located, measured and mapped. The data were plotted in maps of the nests, which served for the simulation of the plans. It was concluded that any of the planting lines tested for the first transect can be used in monitoring the leaf-cutting ants in the study area. The optimum distance between the strip transects is $96 \mathrm{~m}$. The density estimated of leaf-cutting ant nests by the Proportional Area estimator, using the strip transects, is more accurate than by the Cottam\&Curtis estimator, using the line transects, because the first produces results equal to the census. The operational use of the first plan is valid to estimate the total area of leaf-cutting ant nests in the region.
\end{abstract}

Key words: Monitoramento, Formicidae e Eucalyptus spp.

\footnotetext{
${ }^{1}$ Recebido em 17.04.2007 e aceito para publicação em 25.08.2010.

${ }^{2}$ Universidade Federal de Uberlândia, UFU, Brasil. E-mail: <marcelo.areis@hotmail.com>.

${ }^{3}$ Universidade Federal de Lavras, UFLA, Brasil. E-mail: <zanetti@ufla.br>.

${ }^{4}$ Veracel Celulose S.A., VERACEL, Brasil.
} 


\section{INTRODUÇÃO}

No Brasil existem grandes áreas cultivadas com eucalipto para atender à demanda de madeira para diversos fins (AMARAL-CASTRO, 2000). No entanto, plantios extensos e homogêneos favorecem o surgimento de insetos-praga, destacando-se as lagartas desfolhadoras (ZANUNCIO et al., 1998; ZANUNCIO et al., 2001), cupins (MORAES et al., 2002; AMARALCASTRO et al., 2004; ZANETTI et al., 2005) e formigascortadeiras, que são consideradas os principais insetos-praga dos eucaliptais brasileiros (ZANETTI et al., 2000; ZANUNCIO et al., 2002; ZANETTI et al., 2003a), sendo controladas através de combates localizados (ZANETTI et al., 2003b) e sistemáticos (ZANETTI et al., 2003c).

Programas de manejo integrado de formigascortadeiras têm sido implantados para minimizar os problemas causados por essas formigas nos eucaliptais. E um dos grandes desafios é a determinação dos métodos de amostragem que representem a infestação desses insetos nos talhões de eucalipto com precisão adequada (SOSSAI et al., 2005). Por isso, são necessárias pesquisas para desenvolver metodologias de avaliação populacional desses insetos-praga.

Alguns métodos de amostragem de formigascortadeiras foram desenvolvidos, destacando-se as parcelas (CALDEIRA, 2002; REIS, 2005; CANTARELLI et al., 2006) e os transectos em faixa (CALDEIRA, 2002; ZANUNCIO et al., 2002; REIS, 2005; SOSSAI et al., 2005; PINTO, 2006). Esses métodos de amostragem vêm sendo utilizados nos programas de manejo das formigas-cortadeiras, e o erro amostral operacional tem variado entre 10 e $30 \%$, com $95 \%$ de confiabilidade (ZANETTI et al., 2008). No entanto, esses métodos podem não apresentar boa precisão em todas as regiões cultivadas com eucalipto, por isso é necessária a elaboração de planos de amostragem regionalizados, além do desenvolvimento de outros métodos de amostragem, como os transectos em linha.

Este trabalho teve como objetivos desenvolver um plano de amostragem para formigas-cortadeiras pelo método de transectos em faixas, determinar o melhor estimador de densidade populacional de formigueiros para os métodos de transectos em faixa e em linha e validar o melhor desses planos em florestas de eucaliptos, localizadas em áreas de relevo acidentado da Mata Atlântica de Minas Gerais.

\section{MATERIAL E MÉTODOS}

Este trabalho foi realizado em eucaliptais localizados em área de relevo acidentado sob domínio da Mata Atlântica, no Município de Belo Oriente, na região do Vale do Rio Doce, Minas Gerais, no período de janeiro de 2002 a fevereiro de 2005, em cinco talhões contíguos de Eucalyptus urograndis (Eucalyptus urophylla $\mathrm{x}$ Eucalyptus grandis), totalizando 160,22 ha.

Em cada talhão, foi feito um censo populacional dos formigueiros, percorrendo-se cada entrelinha de plantio. Os ninhos foram localizados, georreferenciados e medidos por entrelinha de plantio. A área de terra solta dos ninhos foi estimada multiplicando-se o maior comprimento pela maior largura da área ocupada pelos montes de terra solta (ALVES et al.,1996). Foram gerados mapas em AUTOCAD ${ }^{\circledR}$, contendo o alinhamento do plantio e a posição de cada árvore e de cada formigueiro em cada talhão, os quais serviram para a simulação dos métodos de amostragem.

\subsection{Desenvolvimento do plano de amostragem pelo método de transectos em faixa}

Considerou-se o transecto em faixa uma parcela de duas entrelinhas de plantio de largura e comprimento iguais aos da linha de plantio. Foram simulados os lançamentos de transectos a cada distância múltipla de 24 m, variando de 24 a 240 m e considerando um mínimo de dois transectos para cada uma das distâncias avaliadas por talhão. A influência do número da linha de plantio para o lançamento do primeiro transecto foi testada iniciando em todas as distâncias de transectos citadas acima, nas linhas de plantio 1, 3, 5, 7 e 9 .

Foram estimados o número e área total de formigueiros para cada uma das simulações de lançamento dos transectos em diferentes distâncias e linhas de início, pela fórmula $\mathrm{EP}=\sum_{\mathrm{i}=1}^{\mathrm{n}} \mathrm{Nf}$, em que $\mathrm{EP}$ = estimativa do número ou área de formigueiros por talhão, para cada simulação de transectos; Nf = número ou área de formigueiros por transecto; e $\mathrm{n}$ = número de transectos $(\mathrm{i}=1,2, \ldots, \mathrm{n})$. Além disso, foi feito um censo populacional do número e área total de formigueiros de cada talhão (censo). O número e, ou, a área de formigueiros por talhão, obtidos na amostragem com os transectos, foram correlacionados com os do censo ( $r$-Pearson; $\mathrm{p}<0,05)$. 


\subsection{Comparação dos estimadores de densidade entre os métodos de transectos em faixa e em linha}

Em cada talhão foi calculada a densidade de ninhos por hectare pelo estimador de área proporcional (REIS, 2005), com transectos em faixa, considerando-se a melhor linha para o lançamento do primeiro transecto e a melhor distância entreeles, conforme obtidono item anterior.Afórmula utilizada foi DP (Área Proporcional) $=\left(10000 \times \sum_{\mathrm{i}=1}^{\mathrm{n}} \mathrm{NF}\right) /\left(\sum_{\mathrm{i}=1}^{\mathrm{n}} \mathrm{AT}\right)$, em que DP = número de ninhos/ha; $\mathrm{Nf}=$ número de ninhos por transecto; $\mathrm{AT}$ = área de cada transecto; e n = número de transectos $(i=1,2, \ldots, n)$. Foram considerados os ninhos numa faixa de duas entrelinhas de plantio (Figura 1A).

Nas mesmas faixas usadas para calcular a densidade de ninhos pelo estimador de área proporcional, foi calculada a densidade de ninhos por hectare pelo estimador de Cottam \& Curtis (COTTAM e CURTIS, 1956), com transectos em linha para cada talhão. Foi selecionada a linha de plantio central de cada faixa e medida a distância perpendicular do centro de cada formigueiro até essa linha. A estimativa da densidade de ninhos foi calculada pela fórmula $P_{(\text {Cottam \& Curtis) }}=10000 /\left(\sum_{\mathrm{i}=1}^{\mathrm{n}} \mathrm{M}^{2} / \mathrm{j}\right)$, em que DP = número de ninhos/ha; $\mathrm{j}=$ número de ninhos selecionados $(i=1,2, \ldots, n)$; e $M^{2}=$ média ao quadrado da distância perpendicular de cada ninho até a linha central da faixa (Figura 1B).

Os valores das estimativas de densidade de ninhos pelos dois estimadores e do censo populacional de cada talhão foram submetidos à análise de variância e ao teste de Scott-Knott (pd”0,05). O erro de amostragem (\%) foi calculado pela diferença entre valores observados e estimados de cada variável. A intensidade amostral correspondeu à porcentagem da área amostrada em relação ao total de área do talhão.

\subsection{Validação do melhor plano de amostragem}

Foram selecionados 10 talhões de eucaliptos diferentes dos utilizados para a elaboração dos planos de amostragem. Em cada talhão, os ninhos de formigascortadeiras foram localizados, identificados e medidos. A área de terra solta dos ninhos foi estimada multiplicando-se o maior comprimento pela maior largura da área ocupada pelos montes de terra solta (ALVES et al., 1996). Os ninhos foram classificados por gênero: Atta (sauveiros) e Acromyrmex (quenquenzeiros); e por tamanho: ninhos grandes $\left(>1 \mathrm{~m}^{2}\right)$ e pequenos $\left(<1 \mathrm{~m}^{2}\right)$. Posteriormente,

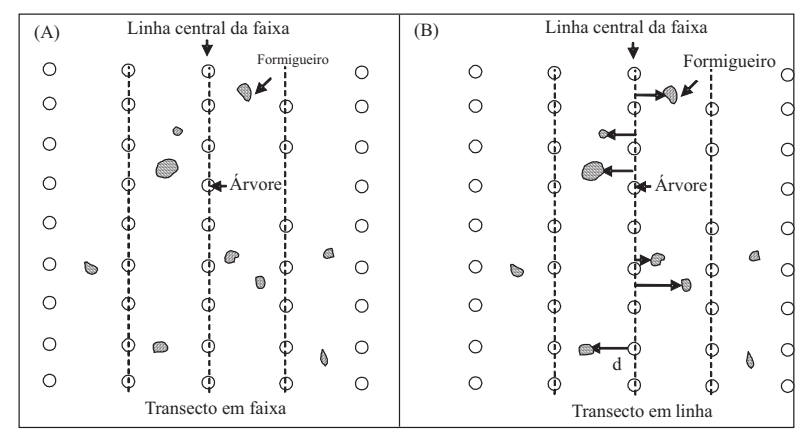

Figura 1 - Esquema mostrando os métodos de amostragem usados para calcular a estimativa da densidade populacional de formigueiros pelo método de transectos em faixa (A) e transectos em linha (B), ambos com duas entrelinhas de largura e comprimento igual ao da linha de plantio.

Figure 1 - Outline showing the sampling methods used to calculate the estimate of the density of the leafcutting ants nest using transects in strip (A) and transects in line $(B)$, both two rows wide and the same length to the planting line.

cada talhão foi amostrado usando-se o melhor plano de amostragem obtido no item anterior. Os valores observados e estimados de cada variável foram submetidos à análise de variância e comparados pelo teste $\mathrm{F}(\mathrm{p}<0,05)$. Calculou-se o erro de amostragem (\%) pela diferença entre valores observados e estimados de cada variável.

\section{RESULTADOS E DISCUÇÃO}

\subsection{Desenvolvimento do plano de amostragem pelo método de transectos em faixa}

Verificou-se que qualquer uma das linhas testadas para o lançamento de primeiro transecto pode ser utilizada no monitoramento de formigas-cortadeiras na área de estudo, pois todas apresentaram correlação significativa entre a população observada e estimada, tanto para a densidade quanto para a área de formigueiros por hectare (Tabela 1). Entretanto, por motivos práticos, selecionou-se a $3^{\mathfrak{a}}$ linha de plantio, pois ela apresentava alinhamento mais regular que a $1^{\mathfrak{a}}$ linha, o que facilitou o caminhamento e a locação das amostras.

Entre as distâncias testadas para o lançamento dos transectos em faixa, apenas as de 24, 48, 96 e 168 $\mathrm{m}$ tiveram valores significativos em todas as linhas de lançamento do primeiro transecto, tanto para densidade quanto para área de ninhos (Tabelas 2 e 3).

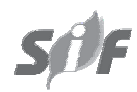

Revista Árvore, Viçosa-MG, v.34, n.6, p.1101-1108, 2010 
Tabela 1 - Correlação entre o censo e a amostragem de formigas-cortadeiras com transectos em faixa, iniciados em diferentes linhas de plantio. Belo Oriente, Minas Gerais.

Table 1 - Correlation between census and the sampling of leaf-cutting ants whit transects in strip, initiate in different planting lines. Belo Oriente, Minas Gerais.

\begin{tabular}{|c|c|c|c|c|c|}
\hline \multirow[t]{2}{*}{ Variável } & \multicolumn{5}{|c|}{ Linha de lançamento do primeiro transecto ${ }^{1}$} \\
\hline & 1 a & $3 \underline{a}$ & 5모 & $7 \underline{a}$ & 9ạ \\
\hline Área de formigueiros $\left(\mathrm{m}^{2} / \mathrm{ha}\right)$ & 0,985 & 0,997 & 0,960 & 0,988 & 0,989 \\
\hline Densidade de formigueiros (n/ha) & 0,996 & 0,998 & 0,997 & 0,998 & 0,989 \\
\hline
\end{tabular}

${ }^{1}$ Todos os valores foram significativos (r-Pearson; $\left.\mathrm{p}<0,01\right)$.

${ }^{1}$ All values were significant ( $r$-Pearson, $p<0.01$ ).

Tabela 2 - Correlação entre o número de ninhos de formigas-cortadeiras observado (censo) e estimado com transectos em faixa por talhão, locados em diferentes distâncias (m) e linhas de lançamento do primeiro transecto (LPT) e intensidade amostral (IA) para cada distância. Belo Oriente, Minas Gerais.

Table2 - Correlation among the number of leaf-cutting ant nests observed (census) and estimates with transects in strip for stands, placed at different distances $(m)$ and release lines of the first transects $(L P T)$ and sampling intensity (IA) for each distance. Belo Oriente, Minas Gerais.

\begin{tabular}{|c|c|c|c|c|c|c|c|c|c|c|c|c|}
\hline \multirow[t]{2}{*}{ Talhão } & \multirow[t]{2}{*}{ LPT } & \multirow[t]{2}{*}{ Censo } & \multicolumn{10}{|c|}{ Número de ninhos estimado por distância entre transectos } \\
\hline & & & $24 \mathrm{~m}$ & $48 \mathrm{~m}$ & $72 \mathrm{~m}$ & $96 \mathrm{~m}$ & $120 \mathrm{~m}$ & $144 \mathrm{~m}$ & $168 \mathrm{~m}$ & $192 \mathrm{~m}$ & $216 \mathrm{~m}$ & $240 \mathrm{~m}$ \\
\hline 433 & $1^{\mathrm{a}}$ & 1702 & 344 & 208 & 113 & 116 & 70 & 82 & 76 & 64 & 36 & 50 \\
\hline 434 & $1^{\mathrm{a}}$ & 5549 & 1140 & 720 & 440 & 320 & 318 & 188 & 198 & 170 & 115 & 134 \\
\hline 435 & $1^{\mathrm{a}}$ & 1056 & 203 & 137 & 74 & 47 & 62 & 36 & 31 & 52 & 39 & 17 \\
\hline 436 & $1^{\mathrm{a}}$ & 2956 & 589 & 321 & 212 & 159 & 153 & 121 & 90 & 93 & 75 & 53 \\
\hline 437 & $1^{\mathrm{a}}$ & 1252 & 231 & 130 & 104 & 61 & 48 & 54 & 48 & 54 & 16 & 21 \\
\hline Correlação* & & 1,00 & 0,99 & 1,00 & 0,99 & 0,99 & 0,98 & 0,98 & 1,00 & 0,96 & 0,98 & \\
\hline 433 & $3^{\mathrm{a}}$ & 1702 & 317 & 222 & 111 & 104 & 65 & 60 & 52 & 79 & 54 & 41 \\
\hline 434 & 3 a & 5549 & 1064 & 570 & 412 & 315 & 240 & 228 & 178 & 197 & 159 & 120 \\
\hline 435 & $3^{\mathrm{a}}$ & 1056 & 238 & 107 & 81 & 60 & 67 & 54 & 25 & 30 & 26 & 21 \\
\hline 436 & $3 \underline{a}$ & 2956 & 557 & 347 & 225 & 182 & 147 & 161 & 98 & 88 & 103 & 62 \\
\hline 437 & $3^{\mathrm{a}}$ & 1252 & 230 & 116 & 105 & 77 & 59 & 52 & 49 & 63 & 37 & 24 \\
\hline Correlação* & & 1,00 & 0,99 & 1,00 & 1,00 & 0,99 & 0,97 & 0,99 & 0,97 & 0,99 & 1,00 & \\
\hline 433 & $5^{\underline{a}}$ & 1702 & 323 & 188 & 144 & 87 & 101 & 77 & 68 & 71 & 28 & 44 \\
\hline 434 & $5^{\underline{a}}$ & 5549 & 1035 & 632 & 431 & 356 & 257 & 187 & 201 & 162 & 154 & 138 \\
\hline 435 & $5 \underline{a}$ & 1056 & 225 & 92 & 91 & 69 & 40 & 48 & 34 & 25 & 22 & 33 \\
\hline 436 & $5 \underline{a}$ & 2956 & 610 & 305 & 254 & 184 & 159 & 138 & 63 & 83 & 120 & 65 \\
\hline 437 & $5 \underline{a}$ & 1252 & 299 & 113 & 98 & 59 & 72 & 46 & 36 & 56 & 29 & 31 \\
\hline Correlação* & & 1,00 & 1,00 & 1,00 & 1,00 & 0,99 & 0,97 & 0,96 & 0,97 & 0,94 & 0,99 & \\
\hline 433 & $7^{\mathrm{a}}$ & 1702 & 323 & 156 & 139 & 95 & 112 & 97 & 59 & 61 & 48 & 42 \\
\hline 434 & 7 a & 5549 & 1166 & 606 & 368 & 356 & 219 & 208 & 218 & 161 & 161 & 118 \\
\hline 435 & $7 \underline{a}$ & 1056 & 215 & 155 & 97 & 57 & 65 & 35 & 41 & 32 & 19 & 25 \\
\hline 436 & $7^{\mathrm{a}}$ & 2956 & 594 & 265 & 222 & 175 & 160 & 106 & 114 & 72 & 103 & 104 \\
\hline 437 & $7 \underline{a}$ & 1252 & 270 & 166 & 77 & 72 & 81 & 82 & 52 & 44 & 19 & 29 \\
\hline Correlação* & & 1,00 & 0,98 & 0,99 & 1,00 & 0,98 & 0,96 & 1,00 & 0,98 & 0,98 & 0,92 & \\
\hline 433 & 9a & 1702 & 395 & 176 & 148 & 85 & 91 & 97 & 53 & 63 & 62 & 67 \\
\hline 434 & $9^{\underline{a}}$ & 5549 & 1144 & 598 & 429 & 330 & 243 & 264 & 161 & 169 & 163 & 128 \\
\hline 435 & $9^{\underline{a}}$ & 1056 & 175 & 135 & 72 & 50 & 53 & 40 & 45 & 40 & 19 & 35 \\
\hline 436 & $9 \underline{a}$ & 2956 & 606 & 308 & 170 & 202 & 195 & 108 & 119 & 106 & 91 & 82 \\
\hline 437 & $9^{\mathrm{a}}$ & 1252 & 222 & 142 & 73 & 84 & 52 & 51 & 77 & 39 & 19 & 16 \\
\hline Correlação* & & & 1,00 & 1,00 & 0,98 & 0,99 & 0,95 & 0,98 & 0,94 & 0,99 & 0,98 & 0,94 \\
\hline IA (\%) & & & 25,0 & 12,5 & 8,3 & 6,3 & 5,0 & 4,2 & 3,6 & 3,1 & 2,8 & 2,5 \\
\hline
\end{tabular}

*Todos os valores foram significativos (r-Pearson; $\mathrm{p}<0,01$ ).

* All values were significant ( $r$-Pearson, $p<0.01$ ).

Revista Árvore, Viçosa-MG, v.34, n.6, p.1101-1108, 2010 
Tabela 3 - Correlação entre a área total de terra solta $\left(\mathrm{m}^{2}\right)$ de ninhos de formigas-cortadeiras observada (censo) e estimada com transectos em faixa por talhão, locados em diferentes distâncias (m) e linhas de lançamento do primeiro transecto (LPT) e intensidade amostral (IA) para cada distância. Belo Oriente, Minas Gerais.

Table 3 - Correlation among the area $\left(\mathrm{m}^{2}\right)$ of leaf-cutting ant nests observed (census) and estimate with transects in strip for stands, placed in different distances $(m)$ and lines of release of the first transects $(L P T)$ and sampling intensity (IA) for each distance. Belo Oriente, Minas Gerais.

\begin{tabular}{|c|c|c|c|c|c|c|c|c|c|c|c|c|}
\hline \multirow[t]{2}{*}{ Talhão } & \multirow[t]{2}{*}{ LPT } & \multirow[t]{2}{*}{ Censo } & \multicolumn{10}{|c|}{ Área total de ninhos estimada por distância entre transectos } \\
\hline & & & $24 \mathrm{~m}$ & $48 \mathrm{~m}$ & $72 \mathrm{~m}$ & $96 \mathrm{~m}$ & $120 \mathrm{~m}$ & $144 \mathrm{~m}$ & $168 \mathrm{~m}$ & $192 \mathrm{~m}$ & $216 \mathrm{~m}$ & $240 \mathrm{~m}$ \\
\hline 433 & $1^{\underline{a}}$ & 4307 & 841 & 497 & 406 & 226 & 131 & 254 & 154 & 146 & 183 & 150 \\
\hline 434 & $1^{\underline{a}}$ & 14983 & 3339 & 1753 & 1460 & 732 & 745 & 460 & 508 & 710 & 312 & 209 \\
\hline 435 & $1^{\mathrm{a}}$ & 4501 & 619 & 860 & 513 & 127 & 296 & 82 & 75 & 147 & 109 & 42 \\
\hline 436 & $1^{\underline{a}}$ & 8947 & 1680 & 923 & 545 & 538 & 285 & 455 & 146 & 173 & 405 & 79 \\
\hline 437 & $1^{\underline{a}}$ & 3200 & 838 & 433 & 335 & 181 & 324 & 248 & 73 & 119 & 29 & 29 \\
\hline Correlação* & & 0,98 & 0,96 & 0,94 & 0,97 & $0,86 *$ & $0,78^{*}$ & 0,93 & 0,92 & $0,75^{*}$ & $0,74^{*}$ & \\
\hline 433 & $3^{\underline{a}}$ & 4307 & 811 & 601 & 308 & 231 & 247 & 73 & 164 & 185 & 55 & 66 \\
\hline 434 & 3 a & 14983 & 2850 & 1528 & 805 & 818 & 615 & 517 & 609 & 500 & 412 & 315 \\
\hline 435 & $33^{\underline{a}}$ & 4501 & 1164 & 266 & 472 & 242 & 163 & 489 & 54 & 55 & 77 & 75 \\
\hline 436 & $33^{\underline{a}}$ & 8947 & 1658 & 759 & 1068 & 537 & 517 & 486 & 170 & 234 & 245 & 199 \\
\hline 437 & 3 a & 3200 & 481 & 306 & 246 & 101 & 94 & 78 & 83 & 377 & 89 & 24 \\
\hline Correlação* & & 0,98 & 0,96 & $0,74 *$ & 0,99 & 0,94 & $0,67 *$ & 0,93 & $0,63^{*}$ & 0,98 & 0,99 & \\
\hline 433 & $5^{\underline{a}}$ & 4307 & 862 & 398 & 248 & 298 & 227 & 170 & 102 & 256 & 106 & 107 \\
\hline 434 & $5^{\underline{a}}$ & 14983 & 3011 & 1536 & 868 & 859 & 550 & 416 & 380 & 329 & 314 & 295 \\
\hline 435 & 5 a & 4501 & 1143 & 549 & 400 & 510 & 155 & 392 & 127 & 201 & 74 & 144 \\
\hline 436 & 5 a & 8947 & 2088 & 1007 & 446 & 583 & 515 & 371 & 107 & 207 & 335 & 378 \\
\hline 437 & $5^{\underline{a}}$ & 3200 & 731 & 446 & 283 & 127 & 296 & 130 & 44 & 122 & 30 & 63 \\
\hline Correlação* & & 0,99 & 0,99 & 0,96 & 0,91 & $0,86^{*}$ & $0,70^{*}$ & 0,91 & $0,78^{*}$ & $0,87 *$ & $0,77^{*}$ & \\
\hline 433 & 7 a & 4307 & 862 & 456 & 324 & 237 & 245 & 408 & 90 & 202 & 181 & 229 \\
\hline 434 & $7 \underline{a}$ & 14983 & 2965 & 1702 & 1028 & 880 & 492 & 453 & 472 & 468 & 399 & 176 \\
\hline 435 & $7 \underline{a}$ & 4501 & 868 & 609 & 263 & 385 & 303 & 166 & 273 & 213 & 27 & 30 \\
\hline 436 & $7 \underline{a}$ & 8947 & 2021 & 744 & 806 & 404 & 435 & 380 & 340 & 272 & 345 & 219 \\
\hline 437 & 7 a & 3200 & 632 & 222 & 149 & 278 & 86 & 219 & 90 & 113 & 19 & 43 \\
\hline Correlação* & & 0,99 & 0,97 & 0,97 & 0,94 & $0,87^{*}$ & $0,70^{*}$ & 0,91 & 0,97 & 0,89 & $0,45^{*}$ & \\
\hline 433 & 9 a & 4307 & 932 & 304 & 295 & 188 & 224 & 250 & 91 & 256 & 163 & 92 \\
\hline 434 & $9^{\underline{a}}$ & 14983 & 2818 & 2102 & 1034 & 809 & 719 & 537 & 481 & 430 & 352 & 528 \\
\hline 435 & $9^{\underline{a}}$ & 4501 & 707 & 502 & 406 & 154 & 182 & 162 & 134 & 141 & 138 & 97 \\
\hline 436 & $9 \underline{a}$ & 8947 & 1500 & 1178 & 403 & 698 & 433 & 304 & 199 & 378 & 170 & 123 \\
\hline 437 & 9a & 3200 & 518 & 296 & 89 & 138 & 138 & 256 & 109 & 71 & 150 & 44 \\
\hline Correlação* & & & 0,99 & 1,00 & 0,94 & 0,94 & 1,00 & 0,92 & 0,97 & 0,88 & 0,93 & 0,94 \\
\hline IA (\%) & & & 25,0 & 12,5 & 8,3 & 6,3 & 5,0 & 4,2 & 3,6 & 3,1 & 2,8 & 2,5 \\
\hline
\end{tabular}

*Valores não significativos (r-Pearson; $\mathrm{p}>0,01)$.

* Values not significant ( $r$-Pearson, $p>0.01$ ).

As melhores correlações foram obtidas pelas distâncias de 24 e $48 \mathrm{~m}$, porém apresentaram custo de amostragem acima de $10 \%$ do custo de controle, o que tornaria a sua execução inviável do ponto de vista econômico. As distâncias de 96 e 168 m apresentaram, também, valores de correlação elevados e intensidade amostral de 6,3 e 3,6\%, respectivamente. Essas intensidades são viáveis economicamente, considerando-se que o custo da amostragem não deve ultrapassar $10 \%$ do custo do controle (Tabelas 2 e 3). No entanto, a amostragem de formigueiros com transectos em faixas a cada $96 \mathrm{~m}$, lançados a partir da terceira linha de plantio, apresentou maiores correlações com o censo na maioria das linhas de início que a distância de 168 m, sendo, portanto, mais indicada para a região.

Estudo semelhante foi desenvolvido em eucaliptais em Montes Claros, Minas Gerais, onde se verificou que o lançamento de transectos em faixa de $9 \mathrm{~m}$ de largura a partir da sétima linha de plantio obteve a melhor correlação com o censo dos formigueiros (SOSSAI, 2001). No trabalho realizado na região de Cerrado, no Município de Bocaiúva, Minas Gerais, 
concluiu-se que a $1^{a}$, $3^{\underline{a}}, 5^{\underline{a}}$, $7^{a}$ ou $9^{\underline{a}}$ linhas de plantio podem ser utilizadas para o lançamento do primeiro transecto. A distância ótima entre elas foi de 96 m, para estimar a área ( $\mathrm{m}^{2} / \mathrm{ha}$ ) e a densidade de sauveiros (n/ha), por apresentarem menor erro de estimativa e implicar menor custo com amostragem. Foi selecionada a quinta linha por ter apresentado maior valor numérico de correlação e ter alinhamento mais regular do que as duas primeiras (CALDEIRA, 2002). Em estudo semelhante também em Montes Claros, constatou-se que o lançamento de um transecto de $9 \mathrm{~m}$ de largura a cada 120 m distância pode ser recomendado para monitorar o número e área de colônias de formigascortadeiras nos eucaliptais estudados (ZANUNCIO et al., 2004).

Da mesma forma como ocorreu com os planos de parcelas ao acaso, os planos de amostragem por transectos são diferentes entre locais, pelos mesmos motivos citados anteriormente. Portanto, eles não podem ser generalizados, e sim regionalizados.

\subsection{Comparação dos estimadores de densidade entre os métodos de transectos em faixa e em linha}

A estimativa da densidade de ninhos de formigascortadeiras pelo estimador de área proporcional usando transectos em faixa foi semelhante ao censo populacional de ninhos, sendo mais precisa do que pelo estimador Cottam \& Curtis usando transectos em linha. Esse último estimador apresentou erro muito elevado em relação ao primeiro, mesmo tendo a mesma intensidade amostral (Tabela 4).

O estimador de área proporcional produziu um erro médio de $1,17 \%$ em relação ao censo, não diferindo estatisticamente deste $(\mathrm{p}>0,05)$, enquanto o estimador de Cottam \& Curtis superestimou a população em $445,80 \%$, diferindo do censo. Essa superestimativa ocorreu, porque o segundo método subestimou a área do transecto quando se limitava a distância máxima de observação (COTTAM e CURTIS, 1956), a exemplo da utilizada neste trabalho ( $3 \mathrm{~m}$ em cada lado do transecto), ou seja, quanto maior a distância dos ninhos à linha de observação, maior a área do transecto e menor a densidade de ninhos por hectare. Isso indica que o estimador de área proporcional deve ser utilizado para calcular a densidade populacional de formigueiros quando se utilizam transectos em faixas de duas entrelinhas de largura, lançados a cada $96 \mathrm{~m}$ a partir da terceira linha de plantio, em programas de monitoramento dessa praga em eucaliptais da região estudada.

\subsection{Validação do melhor plano de amostragem}

O uso de transectos em faixa com estimador de área proporcional, em outra área que não a usada para a elaboração dos planos de amostragem, mostrou que a estimativa da área de sauveiros e quenquenzeiros grandes e da área total de formigueiros foi semelhante ao censo, ao contrário da estimativa de área de sauveiros e quenquenzeiros pequenos (Tabela 5).

O erro médio da estimativa de área de sauveiros grandes por hectare foi de $10,09 \%$, porém com alto coeficiente de variação (346,36\%). No entanto, a estimativa da área de sauveiros pequenos apresentou erro maior do que os sauveiros grandes, semelhante ao verificado na área de quenquenzeiros grandes e pequenos, que apresentaram erros médios de 67,00 e 59,64\%, respectivamente (Tabela 5). Erros-padrão altos nas amostragens de sauveiros pequenos e quenquenzeiros grandes e pequenos significam baixa intensidade

Tabela 4 - Densidade de formigueiros (n/ha) observada (censo) e calculada pelos estimadores de área proporcional com transectos em faixa e de Cottam \& Curtis com transectos em linha, ambos com duas entrelinhas de largura, distanciados a cada 96 m e intensidade amostral (IA) para cada método. Belo Oriente, Minas Gerais.

Table 4 - Density of leaf-cutting ant nests ( $n / h a$ ) observed (census) and estimated by the Proportional Area estimator with transects in strip and by the Cottam\&Curtis estimator with transects in line, both with two row wide distanced to each $96 \mathrm{~m}$ and sampling intensity (IA) for each method. Belo Oriente, Minas Gerais.

\begin{tabular}{|c|c|c|c|c|}
\hline Método & Estimador & Densidade (n/ha) ${ }^{1}$ & Erro (\%) & IA (\%) \\
\hline Censo & - & 78,45 a & - & - \\
\hline Transectos em faixa & Área proporcional & 80,91 a & 1,17 & 6,25 \\
\hline Transectos em linha & Cottam \& Curtis & $428,18 \mathrm{~b}$ & 445,80 & 6,25 \\
\hline
\end{tabular}

${ }^{1}$ Médias seguidas pela mesma letra não diferem entre si (Scott-Knott; p>0,05).

${ }^{1} \mathrm{MED}$ followed by same letter does not differ (Scott-Knott, $p>0.05$ ).

Revista Árvore, Viçosa-MG, v.34, n.6, p.1101-1108, 2010 
Tabela 5 - Área média de ninhos de formigas-cortadeiras observada e estimada pelo estimador de área proporcional com transectos em faixa de duas entrelinhas de largura, distanciados a cada $96 \mathrm{~m}$, erro de amostragem e coeficiente de variação (CV), em 10 talhões cultivados com eucalipto. Belo Oriente, Minas Gerais.

Table 5 - Medium area of leaf-cutting ant nests observed and estimated by Proportional Area estimator with transects in strip of two rows wide distanced at $96 \mathrm{~m}$ each, sampling error and variation coefficient $(C V)$, in 10 eucalyptus stands. Belo Oriente, Minas Gerais.

\begin{tabular}{|c|c|c|c|c|c|}
\hline \multirow[t]{2}{*}{ Variáveis* } & \multicolumn{2}{|c|}{ Área de ninhos (m²/ha) } & \multicolumn{2}{|c|}{ Erro } & \multirow[t]{2}{*}{$\mathrm{CV}(\%)$} \\
\hline & Observada & Estimada & $\left(\mathrm{m}^{2} / \mathrm{ha}\right)$ & $\%$ & \\
\hline Sauveiros $>1 \mathrm{~m}^{2}$ & $123,9 \pm 35,08$ & $111,4 \pm 29,66$ & $12,5 \pm 6,55$ & 10,09 & 346,36 \\
\hline Sauveiros $<1 \mathrm{~m}^{2 *}$ & $96,4 \pm 11,91$ & $44,6 \pm 4,92$ & $51,9 \pm 12,23$ & 53,77 & 47,23 \\
\hline Quenquenzeiros > $1 \mathrm{~m}^{2}$ & $6,7 \pm 2,86$ & $2,2 \pm 0,78$ & $4,5 \pm 2,19$ & 67,00 & 79,26 \\
\hline Quenquenzeiros $<1 \mathrm{~m}^{2 *}$ & $20,7 \pm 3,40$ & $8,4 \pm 1,16$ & $12,4 \pm 2,73$ & 59,64 & 27,64 \\
\hline Total de formigueiros & $247,8 \pm 46,00$ & $166,6 \pm 29,56$ & $81,2 \pm 19,46$ & 32,78 & 35,91 \\
\hline
\end{tabular}

* Variáveis com diferença significativa entre valores observados e estimados (F; p>0,05).

* Variables with significant differences between the observed and estimated ( $F, p>0.05)$.

amostral para esses casos, indicando que o plano de amostragem utilizado não pode ser adotado para estimar, separadamente, a população de formigueiros de cada gênero e tamanho.

O monitoramento de formigas-cortadeiras em florestas em fase de manutenção considera a totalidade de formigueiros independentemente do gênero e do tamanho, para a tomada de decisão. Nesse caso, como a estimativa do total de formigueiros não apresentou diferença significativa com o censo populacional $(\mathrm{p}>0,05)$ (Tabela 5), pode-se considerar que o uso operacional dos transectos em faixa com o estimador de área proporcional é válido para ser utilizado em programas de monitoramente de formigas-cortadeiras na região de estudo.

\section{CONCLUSÃO}

Concluiu-se que qualquer uma das linhas testadas para o lançamento de primeiro transecto pode ser utilizada no monitoramento de formigas-cortadeiras na área de estudo. A distância ótima entre os transectos em faixa é de $96 \mathrm{~m}$. A estimativa da densidade de ninhos de formigas-cortadeiras pelo estimador de área proporcional, usando transectos em faixa, é mais precisa do que pelo estimador Cottam \& Curtis usando transectos em linha, pois o primeiro produz resultados iguais ao do censo. O uso operacional dos transectos em faixa, com o estimador de área proporcional, lançados a cada $96 \mathrm{~m}$ a partir da terceira linha de plantio é válido para estimar a área total de formigueiros na região.

\section{AGRADECIMENTOS}

Ao CNPq e à FAPEMIG, pela concessão de bolsa e auxílio financeiro; e à Celulose Nipo-Brasileira S.A. (CENIBRA), pelo auxílio financeiro e apoio de campo durante a realização deste trabalho.

\section{REFERÊNCIAS}

ALVES, J. B. et al. Métodos de distribuição de isca granulada em formigueiros de Atta laevigata (F. Smith). Revista Árvore, v.20, n.1, p.111-116, 1996.

AMARAL-CASTRO, N. R. Sistema de amostragem e avaliação de danos por cupins de cerne (Insecta: Isoptera) em plantios de Eucalyptus spp. 2000. 97f. Dissertação (Mestrado em Entomologia) Universidade Federal de Lavras, Lavras, 2000.

AMARAL-CASTRO, N. R. et al. Species of soil inhabiting termites (Insecta: Isoptera) collected in eucalyptus plantations in the State of Minas Gerais, Brazil. Sociobiology, v.44, n.3, p.717-726, 2004.

CALDEIRA, M. A. Planos de amostragem de sauveiros em eucaliptais. 2002. $39 \mathrm{f}$. Dissertação (Mestrado em Entomologia) Universidade Federal de Lavras, Lavras, 2002.

CANTARELLI, E. B. et al. Plano de amostragem de Acromyrmex spp. (Hymenoptera:

Formicidae) em áreas de pré-plantio de Pinus spp. Revista Ciência Rural, v.36, n.2, p.385-390, 2006.

Revista Árvore, Viçosa-MG, v.34, n.6, p.1101-1108, 2010 
COTTAM, G.; CURTIS, J. T. The use of distance measures in phytosociological sampling. Ecology, v.37, n.4, p.471-475, 1956.

MORAES, J. C. et al. Effect of Eucalyptus species and soil type on infestion levels of heartwood termites (Insecta: Isoptera) in reforested areas of Brazil. Sociobiology, v.39, n.1, p.145-153, 2002.

PINTO, R. Amostragem e distribuição espacial de colônias de formigas cortadeiras (Hymenoptera: Formicidae) em eucaliptais. 2006. 66f. Tese (Doutorado em Entomologia) - Universidade Federal de Viçosa, Viçosa, MG, 2006.

REIS, M. A. Estudo de métodos aleatório e de distâncias para amostragem de formigas cortadeiras em eucaliptais. 2005. 55f.

Dissertação (Mestrado em

Entomologia) - Universidade Federal de Lavras, Lavras, 2005.

SOSSAI, M. F. Avaliação de métodos de amostragem de formigas cortadeiras em plantios de Eucalyptus spp. 2001.56f. Dissertação (Mestrado em Entomologia) Universidade Federal de Viçosa, Viçosa, MG, 2001.

SOSSAI, M. F. et al. Transects to estimative the number of leaf-cuting ant nests (Hymenoptera: Formicidae) in Eucalyptus urophylla plantations. Sociobiology, v.46, n.3, p.667-676, 2005.

ZANETTI, R. et al. Efeito da densidade $e$ do tamanho de sauveiros sobre a produção de madeira em eucaliptais. Anais da Sociedade Entomológica do Brasil, v.29, n.1, p.105-112, 2000.

ZANETTI, R. et al. Level of economic damage for leaf-cutting ants (Hymenoptera: Formicidae) in Eucalyptus plantations in Brazil. Sociobiology, v.42, n.2, p.433-442, 2003a.
ZANETTI, R. et al. Eficiência de isca formicida aplicada sobre o monte de terra solta de ninhos de Atta sexdens rubropilosa (Hymenoptera: Formicidae). Revista Árvore, v.27, n.3, p.407-410, 2003 b.

ZANETTI, R. et al. Combate sistemático de formigas cortadeiras com iscas granuladas, em eucaliptais com cultivo mínimo. Revista Árvore, v.27, n.3, p.387-392, 2003c.

ZANETTI, R. et al. Estimation of wood volume losses by heartwood termites (Insecta: Isoptera) in eucalyptus plantations in the State of Minas Gerais, Brazil. Sociobiology, v.45, n.3, p.619-630, 2005.

ZANETTI, R. et al. Métodos de amostragem de formigas cortadeiras em florestas cultivadas. In: VILELA, E. V. et al. Insetos sociais: da biologia à aplicação. Viçosa, MG: Universidade Federal de Viçosa, 2008. v.1. p.397-412.

ZANUNCIO, J. C. et al. Influence of strips of native vegetation on Lepidoptera associated with Eucalyptus cloeziana in Brazil. Forest Ecology and Mangement, v.108, n.1/2, p.85-90, 1998.

ZANUNCIO, J. C. et al. Species richness and abundance of defoliating Lepidoptera associated with Eucalyptus grandis in Brazil and their response to plant age. Austral Ecology, v.26, n.6, p.582-589, 2001.

ZANUNCIO, J. C. et al. Spatial distribuition of nests of the leaf-cutting ant Atta sexdens rubropilosa (Hymenoptera: formicidae) in plantations of Eucalyptus urophylla in Brazil. Sociobiology, v.39, n.2, p.231-242, 2002.

ZANUNCIO, J. C. et al. Sampling methods for monitoring the number area of colonies of leaf cutting ants (Hymenoptera: Formicidae) in Eucalyptus plantations in Brazil. Sociobiology, v.44, n.2, p.337-344, 2004. 